\title{
Rare leptonic and semileptonic $b$-hadron decays and tests of lepton flavour universality at LHCb
}

\section{Simone Bifani* ${ }^{*}$}

University of Birmingham, School of Physics and Astronomy, Birmingham, United Kingdom

E-mail: simone.bifani@cern.ch

\begin{abstract}
Rare decays of heavy-flavoured particles provide an ideal laboratory to look for deviations from the Standard Model, and explore energy regimes beyond the LHC reach. Decays proceeding via electroweak penguin diagrams are excellent probes to search for New Physics, and $b \rightarrow s \ell^{+} \ell^{-}$ processes are particularly interesting since they give access to many observables such as branching fractions, asymmetries and angular observables. Recent results from the LHCb experiment are reviewed.
\end{abstract}

38th International Conference on High Energy Physics

3-10 August 2016

Chicago, USA

* Speaker.

${ }^{\dagger}$ On behalf of the LHCb collaboration. 

Fig. 1 (left).

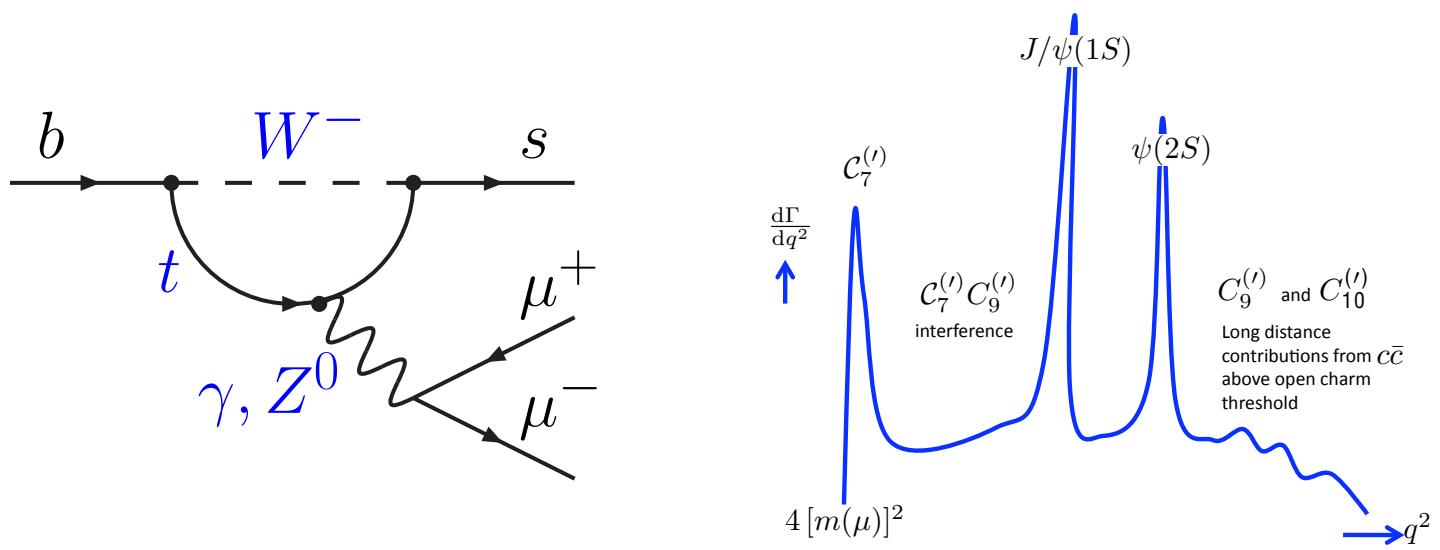

\section{Introduction}

Flavour Changing Neutral Current (FCNC) processes, where a quark changes its flavour without altering its electric charge, are forbidden at tree level in the Standard Model (SM) and can only occur via loop diagrams. This makes such transitions rare and, due to the lack of a dominant treelevel SM contribution, sensitive to new unobserved particles that can show up either as a sizeable increase or decrease in the rate of particular decays, or as a change in the angular distribution of the particles in the detector. A good laboratory to study FCNC are decays of a $b$ quark into an $s$ quark and two leptons, $b \rightarrow s \ell^{+} \ell^{-}$, which are described by the electroweak penguin diagram shown in

The $\mathrm{LHCb}$ detector $[1,2]$ is a single arm spectrometer fully instrumented in the forward region and designed to study heavy-flavoured hadrons. During Run-1, LHCb collected about 1 and $2 \mathrm{fb}^{-1}$ of $p p$ collisions at centre-of-mass energies of 7 and $8 \mathrm{TeV}$, respectively. Due to the large production cross-section in the forward direction these data provide unprecedentedly large numbers of $B$ and $\Lambda_{b}^{0}$ hadron decays. A flexible trigger system, excellent momentum and impact parameter resolutions, and the most performant vertexing and particle identification capabilities at the LHC, make LHCb the ideal place to look for New Physics (NP) through precise studies of rare $b$-quark processes. Recent measurements of semileptonic $b$-hadron decays are discussed.

Figure 1: (left) Electroweak penguin diagram describing the transition of a $b$ quark into an $s$ quark and two leptons. (right) Sketch of the differential branching fraction as a function of the dilepton invariant mass squared, $q^{2}$, for $b \rightarrow s \ell^{+} \ell^{-}$processes. Different Wilson coefficients can be probed in different $q^{2}$ regions.

\section{Branching fractions}

The most basic observable that physics beyond the SM can affect is the rate at which a particular decay occurs, which motivated the LHCb collaboration to perform the measurement of the branching fraction of a series of FCNC processes. All measurements are performed as a function of the dilepton invariant mass squared, $q^{2}$, and compared against the SM prediction. Final states with muons are considered as being experimentally easier to reconstruct. The dependence of the differential branching fraction as a function of $q^{2}$ is shown in Fig. 1 (right). Figure 2 shows the 

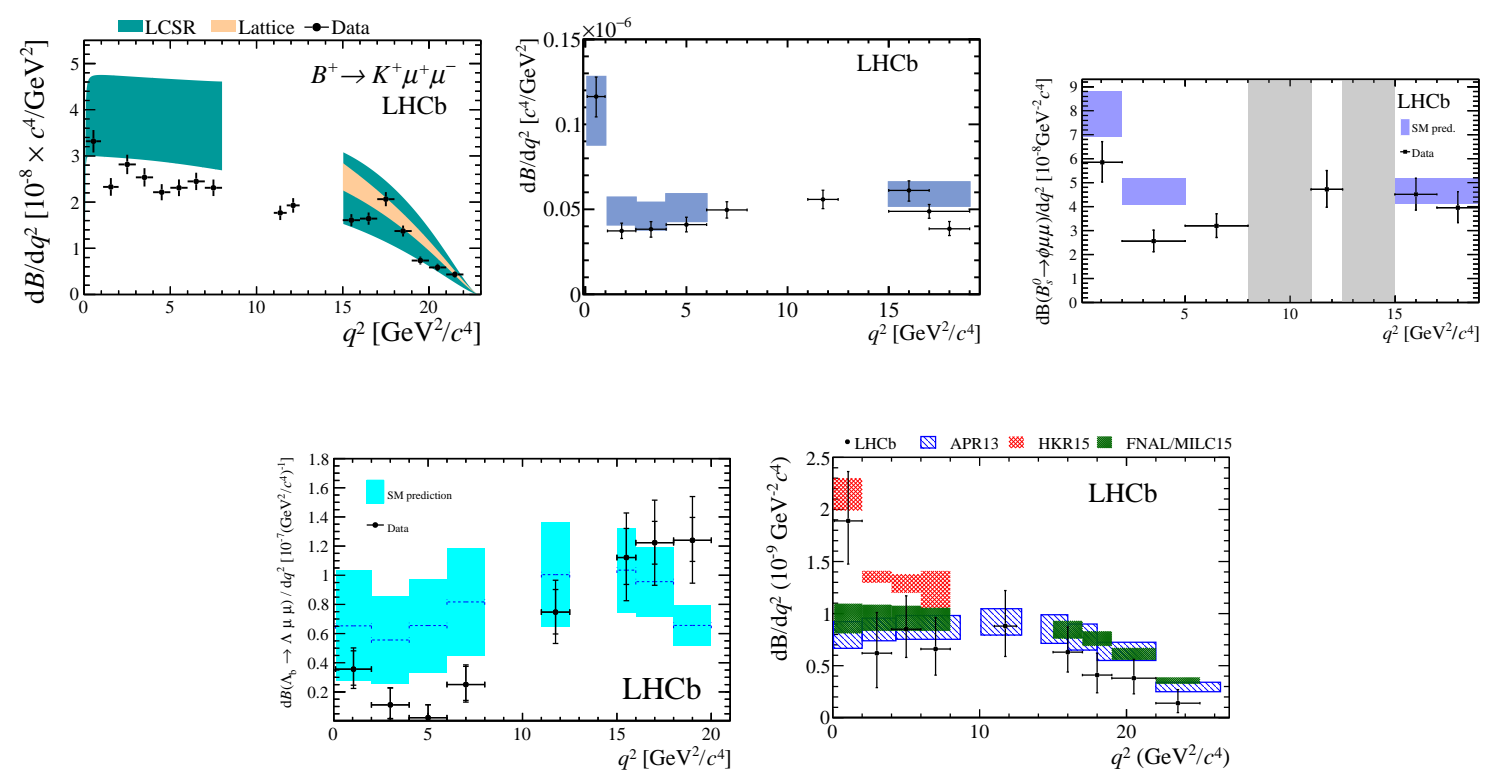

Figure 2: Differential branching fraction of the decay (top) $B^{+} \rightarrow K^{+} \mu^{+} \mu^{-}$[6], $B^{0} \rightarrow K^{* 0} \mu^{+} \mu^{-}$[7], $B_{s}^{0} \rightarrow \phi \mu^{+} \mu^{-}$[3] and (bottom) $\Lambda_{b}^{0} \rightarrow \Lambda \mu^{+} \mu^{-}$[4], $B^{+} \rightarrow \pi^{+} \mu^{+} \mu^{-}$[5], compared to SM predictions.

differential branching fraction of a collection of $b \rightarrow s \ell^{+} \ell^{-}$decays of $B$ and $\Lambda_{b}^{0}$ hadrons, as well as partner transitions such as $b \rightarrow d \ell^{+} \ell^{-}$. In all cases, the experimental uncertainty is dominated by the limited statistics of the samples available in the Run-1 data set.

In the region below $\sim 6 \mathrm{GeV}^{2} / c^{4}$ in $q^{2}$, the $\mathrm{SM}$ predictions consistently overshoot the data, a common trend that is observed both in the mesonic and in the baryonic sectors. The largest deviation is found in the decay $B_{s}^{0} \rightarrow \phi \mu^{+} \mu^{-}$in the region $1<q^{2}<6 \mathrm{GeV}^{2} / c^{4}$, where the data are $3.3 \sigma$ below the prediction [3]. Decays of $\Lambda_{b}^{0}$ hadrons are also overestimated in the SM, however predictions are currently much less precise than for $B$ mesons [4]. Finally, although the $B^{+} \rightarrow \pi^{+} \mu^{+} \mu^{-}$ branching fraction is generally compatible with the prediction, agreement in the lowest $q^{2}$ bin is only achieved when contributions from $\rho$ and $\omega$ resonances are taken into account [5].

\section{Angular analyses}

Besides using branching fractions, much stronger constraints to possible extensions of the SM can be set by studying the angular distribution of the final state particles of FCNC decays. Depending on the decay mode and on the size of the available data sample, full or simplified angular analyses have been performed.

The decay $B^{0} \rightarrow K^{* 0} \mu^{+} \mu^{-}$has a complex angular structure that can be fully described by three angles and $q^{2}$, and that provides many observables sensitive to different types of NP. The LHCb collaboration has performed the first full angular analysis of this mode, and measured the full set of CP-averaged angular terms, their correlations, as well as the full set of CP-asymmetries [7]. The forward-backward asymmetry of the dimuon system, $A_{F B}$, and the longitudinal polarisation fraction of the $K^{* 0}, F_{L}$, compared to the SM prediction are presented in Fig. 3 (top). There is 

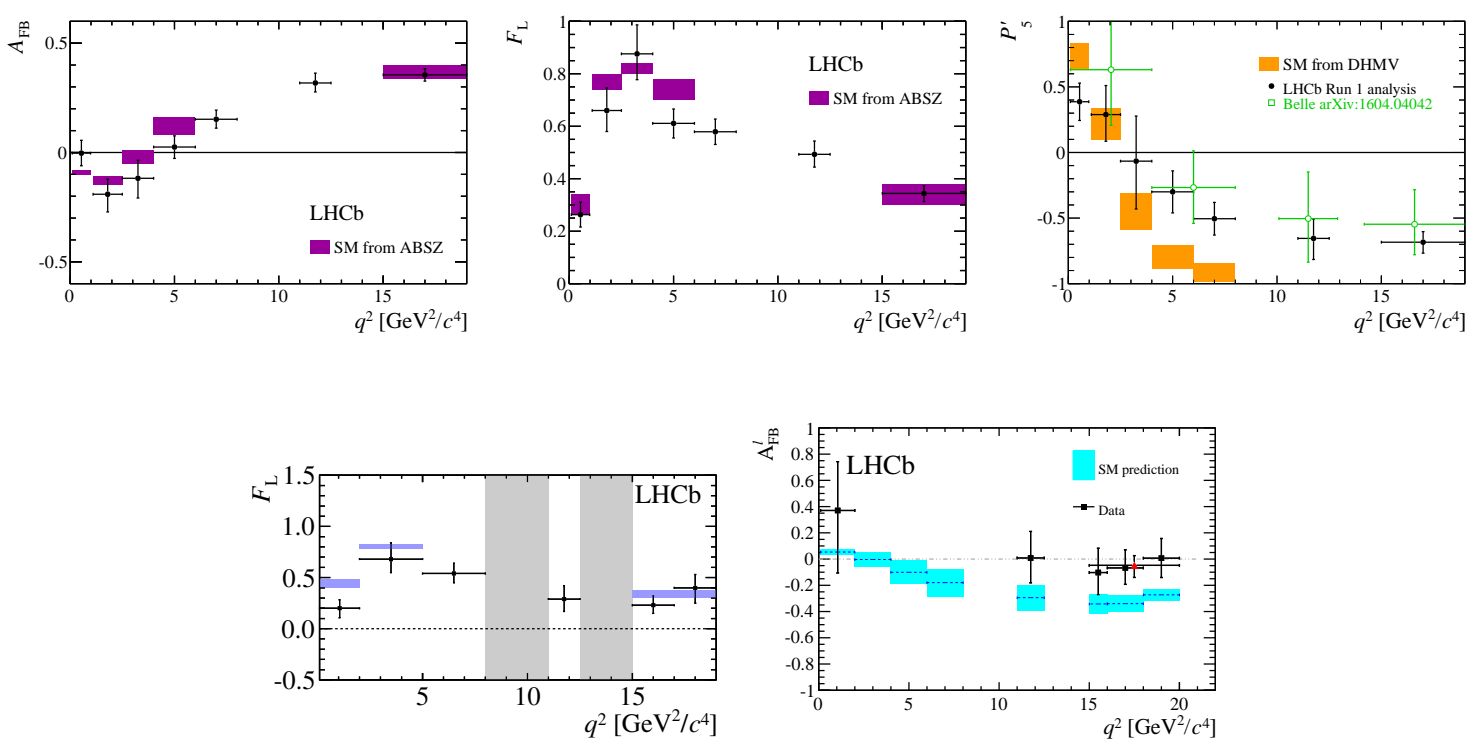

Figure 3: Angular observables of the decay (top) $B^{0} \rightarrow K^{* 0} \mu^{+} \mu^{-}$[7] and (bottom) $B_{s}^{0} \rightarrow \phi \mu^{+} \mu^{-}$[3], $\Lambda_{b}^{0} \rightarrow \Lambda \mu^{+} \mu^{-}$[4], compared to SM predictions.

\section{Tests of lepton universality}

Due to the equality of the electroweak couplings of electrons and muons, the decay rate of processes whose final states only differ by the flavour of the participating leptons are expected to be the same in the SM, except from very small Higgs penguin contributions and difference in phase space due to the lepton mass. In particular, ratios of branching fractions represent a powerful null test of the SM as theoretical uncertainties largely cancel in the predictions, and experimental systematics are much reduced. 
The LHCb collaboration has performed the most precise test of lepton universality using $B^{+} \rightarrow K^{+} \mu^{+} \mu^{-}$and $B^{+} \rightarrow K^{+} e^{+} e^{-}$decay modes to date [10]. In the SM the value of $R_{K}$, defined as $R_{K}=\frac{\mathscr{B}\left(B^{+} \rightarrow K^{+} \mu^{+} \mu^{-}\right)}{\mathscr{B}\left(B^{+} \rightarrow K^{+} e^{+} e^{-}\right)}$, in the range $1<q^{2}<6 \mathrm{GeV}^{2} / c^{4}$ is precisely predicted to be $1 \pm \mathscr{O}\left(10^{-3}\right)$ [11]. The experimental result, displayed in Fig. 4 , is $R_{K}=0.745_{-0.074}^{+0.090}($ stat $) \pm 0.036$ (syst), in tension with the SM prediction at $2.6 \sigma$.

Furthermore, a test involving semileptonic $B$ decays with tau leptons in the final state has been carried out for the first time at a hadron collider [12]. The ratio of branching fractions $R_{D^{*}}=$ $\frac{\mathscr{B}\left(\bar{B}^{0} \rightarrow D^{*+} \tau^{-} \bar{v}_{\tau}\right)}{\mathscr{B}\left(\bar{B}^{0} \rightarrow D^{*+} \mu^{-} \bar{v}_{\mu}\right)}$ is measured to be $R_{D^{*}}=0.336 \pm 0.027($ stat $) \pm 0.030$ (syst), which is $2.1 \sigma$ larger than the value expected in the SM.

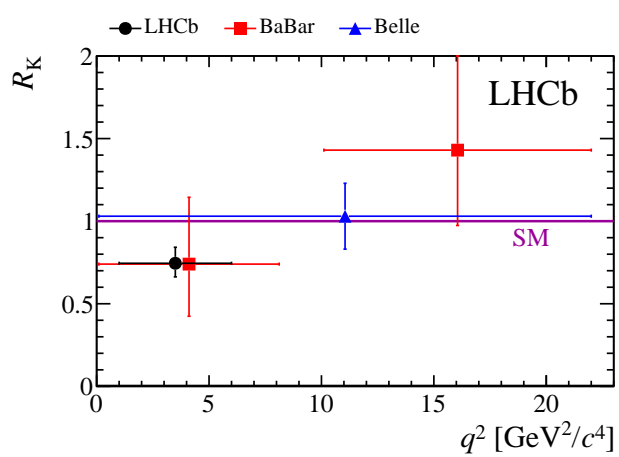

Figure 4: Measurement of $R_{K}=\frac{\mathscr{B}\left(B^{+} \rightarrow K^{+} \mu^{+} \mu^{-}\right)}{\mathscr{B}\left(B^{+} \rightarrow K^{+} e^{+} e^{-}\right)}$[10], compared to previous experiments and to the $\mathrm{SM}$ prediction.

\section{Summary and conclusions}

Recent rare decays of $B$ and $\Lambda_{b}^{0}$ hadrons performed by the LHCb collaboration have been presented. While most of the observables are in good agreement with the SM predictions, some intriguing tensions have been observed, most notably in branching fractions of $b \rightarrow s \ell^{+} \ell^{-}$processes in the low region of $q^{2}$, in the $P_{5}^{\prime}$ angular observable in $B^{0} \rightarrow K^{* 0} \mu^{+} \mu^{-}$, and in $R_{K}$ and $R_{D^{*}}$.

Several attempts to interpret these anomalies have been made by performing global fits to the $b \rightarrow s$ data from different experiments and including various observables [13, 14, 15]. All these fits point to a tension between the data and the SM with a significance of around 3-4 $\sigma$. Various models have been proposed to account for such effects, for example as an indication of a new vector current that would couple more strongly to muons and interfere destructively with the SM vector current $[16,17]$, but a definitive explanation has yet to be found.

The current status strongly motivates further work both in the theory as well as in the experimental side to clarify the present observations. With the upcoming Run-2 data LHCb will continue to perform analyses of rare $b$-quark decays, including additional tests of lepton universality such as $R_{K^{* 0}}$ and $R_{\phi}$.

\section{References}

[1] LHCb collaboration, A. A. Alves Jr. et al., The LHCb detector at the LHC, JINST 3 (2008) S08005. 
[2] LHCb collaboration, R. Aaij et al., LHCb detector performance, Int. J. Mod. Phys. A30 (2015) 1530022, arXiv:1412.6352.

[3] LHCb collaboration, R. Aaij et al., Angular analysis and differential branching fraction of the decay $B_{s}^{0} \rightarrow \phi \mu^{+} \mu^{-}$, JHEP 09 (2015) 179 LHCb-PAPER-2015-023, CERN-PH-EP-2015-145, arXiv:1506.0877.

[4] LHCb collaboration, R. Aaij et al., Differential branching fraction and angular anaysis of $\Lambda_{b}^{0} \rightarrow \Lambda \mu^{+} \mu^{-}$decays, JHEP 06 (2015) 115 LHCb-PAPER-2015-009, CERN-PH-EP-2015-078, arXiv: 1503.0713.

[5] LHCb collaboration, R. Aaij et al., First measurement of the differential branching fraction and CP asymmetry of the $B^{+} \rightarrow \pi^{+} \mu^{+} \mu^{-}$decay, JHEP 10 (2015) 034 LHCb-PAPER-2015-035, CERN-PH-EP-2015-219, arXiv:1509.0041.

[6] LHCb collaboration, R. Aaij et al., Differential branching fractions and isospin asymmetries of $B \rightarrow K^{*} \mu^{+} \mu^{-}$decays, JHEP 06 (2014) 133 LHCb-PAPER-2014-006, CERN-PH-EP-2014-055, arXiv:1403.8044.

[7] LHCb collaboration, R. Aaij et al., Measurement of the $S$-wave fraction in $B^{0} \rightarrow K^{+} \pi^{-} \mu^{+} \mu^{-}$decays and the $B^{0} \rightarrow K^{*}(892)^{0} \mu^{+} \mu^{-}$differential branching fraction, a rXiv: 1606.0473 LHCb-PAPER-2016-012, CERN-EP-2016-141, arXiv:1606. 0473 , submitted to JHEP.

[8] S. Descotes-Genon, T. Hurth, J. Matias, and J. Virto, Optimizing the basis of $B \rightarrow K^{*} \ell^{+} \ell^{-}$observables in the full kinematic range, JHEP 05 (2013) 137, arXiv:1303.5794.

[9] LHCb collaboration, R. Aaij et al., Angular analysis of the $B^{0} \rightarrow K^{*} e^{+} e^{-}$decay in the low- $q^{2}$ region, JHEP 04 (2015) 064 LHCb-PAPER-2014-066, CERN-PH-EP-2014-301, arXiv: 1501.0303.

[10] LHCb collaboration, R. Aaij et al., Test of lepton universality using $B^{+} \rightarrow K^{+} \ell^{+} \ell^{-}$decays, Phys. Rev. Lett. 113 (2014) 151601 LHCb-PAPER-2014-024, CERN-PH-EP-2014-140, arXiv: 1406.6482.

[11] G. Hiller and F. Kruger, More model independent analysis of $b \rightarrow$ s processes, Phys. Rev. D69 (2004) 074020, arXiv:hep-ph/0310219.

[12] LHCb collaboration, R. Aaij et al., Measurement of the ratio of branching fractions $\mathscr{B}\left(\bar{B}^{0} \rightarrow D^{*+} \tau^{-} \bar{v}_{\tau}\right) / \mathscr{B}\left(\bar{B}^{0} \rightarrow D^{*+} \mu^{-} \bar{v}_{\mu}\right)$, Phys. Rev. Lett. 115 (2015) 111803 LHCb-PAPER-2015-025, CERN-PH-EP-2015-150, arXiv: 1506.0861.

[13] W. Altmannshofer and D. M. Straub, Implications of $b \rightarrow s$ measurements, in Proceedings, 50th Rencontres de Moriond Electroweak Interactions and Unified Theories: La Thuile, Italy, March 14-21, 2015, pp. 333-338, 2015. arXiv:1503.0619.

[14] S. Descotes-Genon, L. Hofer, J. Matias, and J. Virto, Global analysis of $b \rightarrow$ sll anomalies, JHEP 06 (2016) 092, arXiv:1510.0423.

[15] M. Ciuchini et al., $B \rightarrow K^{*} \ell^{+} \ell^{-}$decays at large recoil in the Standard Model: a theoretical reappraisal, JHEP 06 (2016) 116, arXiv:1512.0715.

[16] G. Hiller and M. Schmaltz, $R_{K}$ and future $b \rightarrow$ sll physics beyond the standard model opportunities, Phys. Rev. D90 (2014) 054014, arXiv: 1408 . 1627.

[17] D. Ghosh, M. Nardecchia, and S. A. Renner, Hint of Lepton Flavour Non-Universality in B Meson Decays, JHEP 12 (2014) 131, arXiv:1408.4097. 